\title{
Sub-Lamellar Microcracking and Roles of Canaliculi in Human Cortical Bone
}

Vincent Ebacher ${ }^{\mathrm{a}}$, Pierre Guy ${ }^{\mathrm{b}}$, Thomas R. Oxland ${ }^{\mathrm{b}}$, Rizhi Wang ${ }^{\mathrm{a}, *}$

${ }^{a}$ Department of Materials Engineering, University of British Columbia, Vancouver, BC, Canada.

${ }^{\mathrm{b}}$ Department of Orthopaedics, University of British Columbia, Vancouver, BC, Canada.

*Correspondence address:

Dr. Rizhi Wang

Department of Materials Engineering

University of British Columbia

309-6350 Stores Road

Vancouver, BC V6T 1 Z4

Canada

Tel: 1-604-822-9752

Fax: 1-604-822-3619

Email: rzwang@interchange.ubc.ca

DOI: $10.1016 /$ j.actbio.2011.11.013.

(C) 2012. This manuscript version is made available under the CC-BY-NC-ND 4.0 license https:// creativecommons.org/licenses/by-nc-nd/4.0/ 


\title{
Sub-Lamellar Microcracking and Roles of Canaliculi in Human Cortical Bone
}

\begin{abstract}
Bone is a tough biological material. It is generally accepted that bone's toughness arises from its unique hierarchical structure, which in turn facilitates distributed microcracking prior to fracture. Yet, there has been limited progress on the detailed roles of the structural elements in the microcracking process. The present study examines the structure - microcracking relations at the lamellar and sub-lamellar levels of human cortical bone subjected to compressive loading. Laser scanning confocal microscopy revealed a clear influence of the local structure and porosity of the Haversian systems' lamellae on microcrack development. In particular, crack initiation and growth under transverse compression were associated with stress concentration at canaliculi. Later stages of microcracking showed extensive sub-lamellar cracks forming cross-hatched patterns and regularly spaced 0.5 to $1.7 \mu \mathrm{m}$ apart. The density, size and regularity of the crack patterns suggest enhanced inelastic deformation capacity through cracking control at the level of mineralized collagen fibril bundles. The present study thus improves the current understanding of the nature of inelastic deformation and microcracking in bone and further suggests that bone's resistance to fracture is achieved through microcrack control at multiple length scales.
\end{abstract}

Keywords: Bone fracture, Cortical bone, Inelastic deformation, Microcracking, Canaliculi, Confocal microscopy 


\section{Introduction}

Bone is a nanocomposite of carbonated apatite nanocrystals and organic phases mainly composed of fibrous collagen protein. It is known for its unique hierarchical structure which has been hypothesized to give rise to its exceptional mechanical performance [1-4]. Although highly mineralized and with an extensive porosity network, human cortical bone exhibits remarkable inelastic deformation [4-8]. Such deformation is critical to its resistance to fracture as it relaxes stress concentrations [8-12]. However, how bone's different structural levels, from the mineralized collagen fibrils to the Haversian systems (or secondary osteons) and their lamellar organization, are involved in the deformation process is still poorly understood.

Bone's inelastic deformation at nano-scale has been associated with slip at mineral/collagen interfaces [7,13], increased energy dissipation arising from nanostructural heterogeneities [14], interfibrillar shear sliding [15-17], and unfolding of non-collagenous proteins (NCPs) [18,19]. At the micro-scale, the inelastic deformation is accompanied by distributed microcracks [6,20] which, in human cortical bone, are present within both interstitial and osteonal lamellae [21,22]. Microcrack initiation has been linked to Haversian canals [21,23] and osteocyte lacunae [24,25]. Long microcracks $(\sim 100 \mu \mathrm{m})$, forming at later stages of cracking [26], interact with osteons [27] and the hyper-mineralized [28] cement lines [29,30]. "Bridges" at various length scales also hinder crack growth $[11,31,32]$. Despite these progresses, the nature of microcracking (distributed microcracks) at the lamellar, sub-lamellar, and fibrillar levels is still largely unknown $[8,33,34]$.

Our recent study has shown the role of the osteonal lamellae in redistributing stress around each Haversian canal through the stable development of multiple intralamellar microcracks [8]. However, where those cracks initiated and how they developed within the bulk remained unclear [8,34]. Canaliculi are fine channels $(\sim 200 \mathrm{~nm})$ [35] connecting the osteocyte lacunae together. Despite their high distribution density $\left(1 \times 10^{6}\right.$ canaliculi $\left.\mathrm{mm}^{-3}[1]\right)$ and potential as stress concentrators [36], their roles in the microcracking process remain hypothetical [37,38]. A detailed study of bone's microcracking process would clarify the relations between microcracks and those fine structures. 
The present microscopy study focused on the structure - microcracking relations at the lamellar and sub-lamellar levels of human cortical bone. The purpose was to investigate the morphology and the development of microcracks within the osteonal lamellar structure and with respect to the canaliculi network. This was carried out through imaging with a laser scanning confocal

microscope following longitudinal and transverse compressive loading. The work is the first step to bridge the gap between microcracking and nano-scale deformation mechanisms. It provides insight into the design strategies used in bone to resist fracture.

\section{Materials and Methods}

The present study investigated the microcracking process under compression in seventeen human

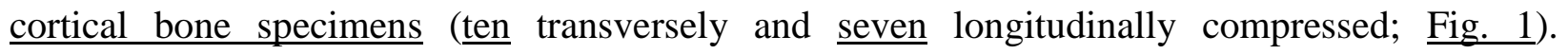
Mechanical characteristics and initial fluorescence microscopy analyses have been reported previously [8,34]. Figure 1 summarizes the experimental procedures described in details in the following sections. All studies were approved by the Clinical Research Ethics Review Board at the University of British Columbia.

\section{Specimens and Mechanical Testing}

As formerly described [8,34], the seventeen cortical bone specimens were extracted from three unembalmed human cadaveric femoral shafts taken from healthy donors (2 males, 64 and 69 years old; 1 female, 55 years old), free from metabolic bone diseases. They were mechanically ground (Beta, Buehler) into rectangular prisms, polished on all surfaces using diamond suspensions down to $1.0 \mu \mathrm{m}$, and vibration polished (Vibromet 2, Buehler) using $0.05 \mu \mathrm{m}$ colloidal silica suspension down to their final dimensions of either $3 \mathrm{~mm} \times 3 \mathrm{~mm} \times 6 \mathrm{~mm}$ or 2.5 $\mathrm{mm} \times 2.5 \mathrm{~mm} \times 5 \mathrm{~mm}$. The specimens were then loaded under monotonic (fourteen specimens) or step-wise (three specimens) compression (Instron 8874, $25 \mathrm{kN}$ load cell or Minimat Materials Tester 2000, $1 \mathrm{kN}$ load cell) at a crosshead speed of $0.1 \mathrm{~mm} / \mathrm{min}$ under wet conditions (Fig. 1). For each orientation, two specimens were loaded to fracture and five specimens were unloaded before fracture (i.e., at about 5\% drop from peak load) to better analyze microcracking. The 
extent of damage was assessed by counting the proportions of damaged osteons from the middle third of the latter specimens.

\section{Laser Scanning Confocal Imaging Analysis}

Following mechanical testing, all specimens were stained with fluorescein (Fisher Scientific) in order to label the microcracks (Fig. 1). The dye also stained the specimens' original surfaces and porosity network. Sixteen (nine transverse and seven longitudinal) specimens were dehydrated and stained according to a procedure described elsewhere [21]. Briefly, the specimens were sequentially immersed into acetone and a series of ethanol/water solutions $(80 \%, 90 \%$, and $100 \%$ ) for periods of 24 hours per step, followed by overnight staining under vacuum in a filtered saturated solution of fluorescein and $70 \%$ ethanol. They were then taken through various preparation and imaging steps to characterize the microcracking. The remaining specimen was stained for 30 minutes in an aqueous solution of $2 \%$ fluorescein, rinsed 10 minutes in tap water, and directly taken to LSCM for examination under water immersion (both specimen and objective lens) in order to verify if secondary staining occurred during subsequent preparation steps.

Following staining, all specimens were examined under the optical microscope using reflected white light (BF; Nikon Eclipse LV100) as well as epi-fluorescence light (EF; Nikon Eclipse E600) with excitation at approximately $490 \mathrm{~nm}$ and emission at approximately $525 \mathrm{~nm}$ (Fig. 1). Four transversely compressed specimens were ground and polished to their central sections (two were ground normally to the osteons and two parallel to the osteons) to characterize microcracking within the bulk. Specific sites were imaged at higher magnification under the laser scanning confocal microscope (LSCM; Olympus FluoView FV1000, Olympus Canada Inc., Markham, Canada). Three specimens were sputter-coated with a thin layer of gold for scanning electron microscope (SEM; Hitachi S-3000N and/or Hitachi S-4700, Hitachi Ltd., Tokyo, Japan) examination. These steps (Fig. 1) allowed for the description of microcracks' morphology and development in relations to bone's hierarchical structures (osteons, osteonal lamellae, osteocyte lacunae, canaliculi, etc.). 
LSCM imaging was carried out under oil immersion (objective lens) while the specimens, resting on the cover slide of an in-house built chamber, were immersed in pure ethanol (Commercial Alcohols Inc.) with the exception of one specimen which was immersed in water (see above; Fig. 1). The $488 \mathrm{~nm}$ line of a multi-line Argon laser was used for excitation and the specimens emitted at $519 \mathrm{~nm}$. The z-step (z-axis defined as the image depth) was set at $200 \mathrm{~nm}$ (resolution in $\mathrm{z}$ of approximately $587 \mathrm{~nm}$ ) and the specimens were typically imaged to a depth of $20 \mu \mathrm{m}$ starting at the surface. A $20 \mu \mathrm{m}$ z-stack thus consisted of a series of 100 images, each $200 \mathrm{~nm}$ apart (but capturing light from $587 \mathrm{~nm}$ thick planes), to a depth of $20 \mu \mathrm{m}$. Hence, threedimensional (3D) information of the cracking and the porosity network was obtained. All measurements were calibrated based on $200 \mathrm{~nm}$ diameter fluorescent microspheres images (TetraSpeck microspheres $0.2 \mu \mathrm{m}$, Molecular Probes Inc.).

All Z-stack images were reviewed qualitatively for microcracks' shape and approximate dimensions. As the canaliculi were suspected to influence transverse compression microcracking, cracks and canaliculi distributions were analyzed using ImageJ 1.43s (National Institutes of Health; USA). Sub-lamellar crack spacing was measured in fourteen damaged regions from $\underline{\text { seven different specimens (two regions or images per specimens). Similarly, fourteen other }}$ regions were used to characterize the canaliculi distribution. Canaliculi density was obtained by counting the intensity peaks of profiles plotted along three lines on $15 \mu \mathrm{m}$ z-stack images (accumulation of virtual slices displayed as one slice; e.g., Fig. 4a) and dividing the count by the volume subtended by each line. Canaliculi spacing was obtained by digitally measuring the distance between the intensity peaks along the same lines, but for a single thin plane of approximately $587 \mathrm{~nm}$ (approximate resolution in z). Canaliculi volume fraction (vol\%) was estimated based on their averaged measured density and reported diameter [35]. Cracks and canaliculi spacings were compared using a t-test (Primer of Biostatistics version 6.0; McGrawHill Companies, USA) with a confidence level of $95 \%(\mathrm{p}<0.05)$. 


\section{Results}

Multi-Scale Cracking in Bone: The Advantages of LSCM

\section{Transverse Compression Cracking}

Transverse compression loading resulted in a fracture plane oblique to the loading direction and along the length of the osteons (Figs. 2a-b). At the osteonal-interstitial level, extensive crosshatched damage ( $80 \pm 5 \%$ of osteons were damaged) was homogeneously distributed within the bulk (Fig. 2c). At the level of a single osteon (Figs. 2d-e), each set of cross-hatched cracks consisted of groups of intralamellar (within the bright/thick layers where fibrils are predominantly longitudinally aligned [39-41]) arc-shaped microcracks radiating out into the quadrants along oblique directions from the Haversian canal, as reported previously $[8,34]$. Under the fluorescence microscope $(\mathrm{EF})$ where background light compromises resolution, arcshaped microcracking appeared as single, blurry cracks inside each lamella (Fig. 2f). Very interestingly, the damage inside each arc-shaped microcrack was resolved into finer cracks under the LSCM (Fig. 2g). These intralamellar microcracks also formed a cross-hatched pattern (Fig. $2 \mathrm{~g}$ ) composed of relatively long circumferentially oriented cracks, $0.9 \pm 0.2 \mu \mathrm{m}$ (range: 0.7 to 1.7 $\mu \mathrm{m}$ ) apart, and radially oriented cracks, $0.8 \pm 0.1 \mu \mathrm{m}$ (range: 0.5 to $1.2 \mu \mathrm{m}$ ) apart, with lengths ranging from below $1 \mu \mathrm{m}$ up to about $10 \mu \mathrm{m}$.

The detailed morphology was generally similar from one osteon to another. The intralamellar microcracking varied in the extent of circumferential and radial cracking and the orientation of radial cracks. Dark layer damage (e.g., Fig. 4e) and flame-like cracking at the ends of circumferential microcracks were also often observed (Supplementary Material Fig. S1). Those variations occurred due to either the stage of damage development or slight changes in the local stress field (e.g., location within the shear band, events at the crack tip, near-surface effects, etc.). Water immersion LSCM imaging of the fresh (non-dehydrated and stained in an aqueous fluorescein solution) specimen showed the same features as dehydrated specimens subjected to post-staining preparation steps (Fig. 1). All microcracking morphologies of transversely compressed bone (presented in Figs. 2, 4, and S1) were thus non-artifactual. 


\section{Longitudinal Compression Cracking}

The fracture following longitudinal compression was oblique to the osteons (Figs. 3a-b). At the osteonal-interstitial level, the well-known cross-hatched microcracks were distributed in $69 \pm 5$ $\%$ of the osteons (Fig. 3c). Higher magnification observations revealed interactions with lamellar and sub-lamellar structures. Within the osteons, cross-hatched microdamage consisted of relatively long microcracks developing oblique to the lamellae (Fig. 3d). Although roughly linear, those cracks locally changed orientation between layers in a "stairway-like" manner (Fig. 3d), being more parallel to lamellar boundaries (and loading direction) within dark layers where fibrils are oriented along the osteons [40-42]. Uncracked ligaments/bundles (and lamellae), previously reported in the literature [11,31,32,37], were also mostly observed within these layers (Fig. 3d insert). High resolution LSCM images (Figs. 3e-g) further showed that the microcracks consisted of many smaller cracks, particularly near the stairway steps and the osteonal-interstitial boundary where the cracking morphology changed to arrays of small wavy and less oblique cracks spreading into a wider area ahead of the main crack tip. Further down in scale, longer microcracks near the Haversian canals would sometimes locally develop finer cracks (Fig. 3f), similar in size and spacing to those of transversely compressed bone. The microcracks also interacted with osteocyte lacunae (Fig. 3g), consistent with literature [24,25,43], and there were evidences of crack interactions with canaliculi which, in some cases, may have developed small cracks (Fig. 3g).

Bone lamellae influenced the microcrack paths and morphologies in specimens compressed along both orientations (Figs. 2e-g and 3d-e). However, at the resolution of LSCM, the structure - microcracking relations in longitudinally compressed specimens were not as easily recognizable (e.g., Fig. 3f) as in transversely compressed specimens. Therefore, as a first step to understand those relations, the latter group was further examined.

\section{Bone's Porosity: The Canaliculi Network}

Fluorescein also stained the densely distributed canaliculi network. As seen on a z-stack LSCM image of an intact osteon, the canaliculi distribution is uniform (Fig. 4a). The average canaliculi spacing was $4.3 \pm 1.4 \mu \mathrm{m}$ and, in agreement with literature [1], the average osteonal canaliculi 
density was $1.6 \pm 0.8 \times 10^{6}$ canaliculi $/ \mathrm{mm}^{3}$. This would correspond to a volume fraction of about $5.0 \%$.

\section{Sub-Lamellar Crack Initiation and Development}

Although most osteons in transversely compressed specimens were damaged, they were at various stages of damage development. Even different quadrants of an osteon showed different stages (Fig. 2e). This allowed for the study of crack initiation and development. Figure 4a shows an intact osteon. Figures $4 \mathrm{~b}$ to $4 \mathrm{e}$ present the cracking sequence within the bulk from early initiation to a late stage corresponding to shear band formation.

First, small circumferential cracks initiated at the canaliculi (Figs. 4b-c) within the bright layers of each osteonal quadrants. Interestingly, multiple crack nucleations not only occurred at numerous canaliculi but also along the length of a single canaliculus (Fig. 4b). Although also initiating cracks [8], osteocyte lacunae are far fewer $\left(1.5 \times 10^{4} \mathrm{~mm}^{-3}\right.$ [1] $)$ than canaliculi and thus did not seem to play a critical role in the process. Following initiation, the cracks merged together forming longer circumferential cracks. As the main crack propagated, other cracks initiated at canaliculi ahead of the main crack tip and eventually merged with it (Fig. 4d). A similar merging mechanism also took place in the depth direction (Fig. 4d z-plane). Hence, the micro-scale arc-shaped cracks, formed from numerous finer cracks (Fig. 4e z-plane), penetrated deep into the lamellar structure taking a "sheet-like" (long but relatively flat) appearance that followed the curvature of the lamellar boundaries. Longitudinal sections' imaging also supports these observations indicating that crack growth also happened parallel to the osteons. As a single canaliculus could initiate many cracks, circumferential cracking usually occurred on more than one plane resulting in many parallel, but not necessarily independent (linked through the depth and/or via radial cracking), circumferential cracks. During the development of circumferential cracks, radial cracking also occurred and linked the circumferential cracks together (Figs. 2e-g and $4 \mathrm{e}$ ). Those radial cracks differed from the radially oriented canaliculi. Their high distribution density could not be explained by the larger canaliculi spacing. This suggests a relation to the mineralized collagen fibrillar bundles. The next stage of damage development was less clear but could involve lengthening and widening of sub-lamellar cross-hatched (Figs. 2e and 4e). At 
certain points, the damaged bright layers could not provide adequate support to the dark layer fibrils. This culminated into shear band formation at the osteonal level [8] through kink failure resulting in damage within the dark layers and a radially oriented microcrack at the Haversian canal (Fig. 4e).

\section{Discussion}

In the present study, LSCM following compressive loading enabled high resolution 3D imaging of sub-lamellar cracks within the bulk of human cortical bone (Figs. 2e-g and 3e-f-g). Transverse compression proved particularly valuable to examine the structure - microcracking relations at the lamellar and sub-lamellar levels. Multiple intralamellar cracks progressively developed within the osteonal wall (Figs. 2c-d-e). The initial stages involved multiple nucleations and crack coalescence through the canaliculi (Figs. 4b-c-d). Further, the process resulted in extensive sublamellar cross-hatched cracks regularly spaced 0.5 to $1.7 \mu \mathrm{m}$ apart (Fig. $2 \mathrm{~g}$ ), a size comparable to mineralized collagen fibril bundles. The high crack density, also observed under longitudinal compression (Fig. 3f), suggests cracking control at the fibrillar level. Such fine damage could further stabilize microcrack growth at higher hierarchical levels. The findings hint at the nature of cracks in bone and the roles of canaliculi in bone's mechanical and remodeling responses.

\section{Role of Canaliculi in Crack Initiation and Growth}

Material failures are generally associated with high local stresses and strains around defects. In bone, high strains and microcrack initiation have been linked to Haversian canals [21,23] and osteocyte lacunae $[24,25,44]$ while the roles of the highly populated canaliculi (Fig. 4a) have not been demonstrated. As illustrated in Figure 5a, the present study showed that in the bulk of transversely compressed bone, multiple short circumferential cracks initiated at the canaliculi (Figs. 4b-c). Those cracks were located within the high interlamellar shear zones (Fig. 5a; shear normal and parallel to the lamellae, i.e., along and across the canaliculi) surrounding the Haversian canals [8]. They eventually merged together (Fig. 4d), by a process similar to shear micro-voids coalescence in metals [45], and developed into long, roughly parallel cracks along both the circumferential and longitudinal (depth) osteonal directions (Fig. 5a; y and z directions, 
respectively). A 3D mechanical simulation (ABAQUS/CAE Version 6.7-5; Dassault Systèmes) of a canaliculus under pure shear revealed 1.4 times increases in local elastic stresses and strains along the canaliculi z-axis (depth direction parallel to osteons) and spanning their entire length. Weak sites at these locations would likely initiate cracking. This corresponds well with the crack initiation observations (Fig. 4c) and supports the merging process along the depth direction (Fig. 4d). Note that the process described here within the bulk is different from near-surface events where radial cracks develop into circumferential microcracks [8]. Although based on many repeated observations, the sequence presented also assumed that the intralamellar microcracking morphological variations represented different stages of the same process. Such an assumption was necessary in view of the technical difficulties (e.g., events happening within the bulk, necessity to label microcracks, resolution of LSCM with water immersion, photobleaching, etc.) associated with monitoring the progress of individual microcracks.

Longitudinal compression seemed less sensitive to canaliculi (rather being more sensitive to osteocyte lacunae (Fig. 3g) [25] and Haversian canals [21]) as few possible cases of canaliculi crack initiation were found (Fig. 3g). Nonetheless, considering their orientation [36], the canaliculi may have similar roles in other loading modes. They have been suspected to be involved in fracture under longitudinal tension [37] and to interact with diffuse damage in human bone [38]. Torsion loading, for which inelastic strains have been associated with circular cracking [46], introduces a similar interlamellar shear state within the osteonal wall (Fig. 5a) and hence is likely to involve canaliculi. Pre-existing microcracks, generally attributed to in vivo fatigue $[47,48]$, are thought to contribute to fragility and stress fractures [49,50]. Those were examined in our previous study [8]. Upon re-visiting the BF and EF images, more than half were found to be parallel to interstitial lamellae boundaries, consistent with others' observations $[29,48]$. Although difficult to verify, this suggests that canaliculi may be similarly involved in initiation and growth of microcracks in vivo.

\section{Possible Role of Canaliculi in the Remodeling Process}

One characteristic that distinguish human cortical bone from other hard tissues such as dentin is its ability to adapt to mechanical loads through remodeling [1,4]. High strains [44,51] and 
microcracks [25,47,52-55] have been proposed as stimuli for mechanosensation through disturbances of lacuno-canalicular network fluid flow [56-59] and/or osteocyte syncitium integrity $[55,58,60]$. Long microcracks have been shown to rupture the osteocyte processes within the canaliculi network [61]. Interestingly, a recent study showed that there is a decrease in osteocyte lacunar density in aged human bone and proposed that this may cause deteriorations in the canalicular fluid flow, reduce the detection of microdamage, and lead to failure and delay of remodeling [62]. Our results support these theories. We hypothesize that canaliculi are directly involved in the remodeling process through initiating microcracking. As cracking at the canaliculi would disturb the lacuno-canalicular fluid flow or rupture the osteocyte processes, the canaliculi, with their uniform distribution and high density (Fig. 4a) [1], would indeed be wellorganized for damage detection. According to the canaliculi spacing results, any crack longer than about $6 \mu \mathrm{m}$ (consider the size of a single crack in tensile "diffuse" damage) would encounter a canaliculus. This may thus represent an early damage detection strategy used by osteocytes to activate the remodeling response. In the aging population where the osteocyte lacunar density is low [62], the canalicular density would be decreased accordingly. This could lead to deficient damage detection, excessive microcrack accumulation, and increased bone fragility.

Sub-Lamellar Cracking: Towards the Nature of Cracks in Bone

All cracks start at bone's lower hierarchical levels. Recent studies have shown the involvement of mineral/collagen interfaces [7,13], NCPs [18,19,32], nano-scale heterogeneity [14], and mineralized collagen fibrils [15-17] in bone's deformation process. It is challenging to relate microcracks to such fine structures. LSCM has proven a powerful technique to investigate submicroscopic bone cracks and porosity [20,33,38,63]. Its resolution approaches the size of the mineralized collagen fibrils. For both loading orientations, the present LSCM study revealed a clear sub-lamellar cross-hatched pattern involving high density of very fine cracks (Figs. $2 \mathrm{~g}$ and $3 f)$.

Transverse compressive loading showed a particularly clear relation with the local structure of each lamella. Extensive cracking developed intralamellarly within the osteonal bright layers (Figs. 2d-e-g). Considering the local fibrillar orientation parallel to the osteons, the regular crack 
spacing, which was smaller than the canaliculi spacing, suggests that a repeating structure of about $800 \mathrm{~nm}$ in size, such as the mineralized collagen fibril bundles, would govern the cracking. The radial cracks are reminiscent of matrix cracks in $2 \mathrm{D}$ fiber-reinforced composites under interlaminar shear [9]. This similarity explains their intralamellar confinement. The cracks evolved without significant fibril interactions in the bright layer while fibrils impeded their growth (e.g., by bridging [31,32]) near the dark layer. The size and regularity of the sub-lamellar cross-hatched pattern further suggest an interfibrillar (as opposed to intrafibrillar) nature, akin to interfacial and matrix shear cracking in transversely compressed fiber-reinforced polymers [64]. This interpretation was proposed based on SEM observations of fractured trabecular bone [32]. It is also well-supported by TEM observations following single osteon loading [37,65]. Such scenario, illustrated in Figure 5b, would likely involve NCPs $[18,19,32,66]$ and extrafibrillar minerals/proteins interfaces $[12,67]$. Further work is planned to directly link sub-lamellar cracks to the mineralized collagen fibrils and to consider other loading modes.

Mechanically, such high crack density could enhance the inelastic strain capacity of the lamellae (consider the strain in a solid containing many microcracks as compared to just a few [68]), further stabilizing the microcracking process at higher hierarchical levels. Interestingly, long interstitial microcracks, such as those pre-existing cracks commonly found in vivo $[50,69,70]$, often extend to the osteons [27,29,49] and larger cracks usually demonstrate a process zone with damaged osteonal lamellae [21,38,71]. Intralamellar microcracking may be another mechanism (along with crack deflection [30] and bridging [31,32]) used in bone to hinder interstitial microcrack growth. Such strategy could provide both robustness to the material and stimulus for remodeling.

\section{Conclusions}

High resolution laser scanning confocal microscope observations of compressed human cortical bone enabled us to establish relations between microcracking and the osteons' lamellar and sublamellar structures. The numerous canaliculi initiated and facilitated the development of multiple intralamellar cracks. Such association implies stress concentration at canaliculi but may also be a strategy for early damage detection. The later stages of the microcracking process involved high 
densities of sub-lamellar cracks forming regular cross-hatched patterns. Those patterns consisted of very fine cracks only a few microns in length and less than $1 \mu \mathrm{m}$ apart. Their size and regularity could result from crack control at the level of the mineralized collagen fibrils. The present findings thus suggest that fracture resistance in bone is achieved by controlling microcrack development at multiple hierarchical levels. The study advances our knowledge on the nature of microcracks in bone and their link to the mineralized collagen fibrils. 


\section{Acknowledgements}

This study was supported by the Canadian Institutes of Health Research and the Michael Smith Foundation for Health Research. R. W. is incumbent of the Canada Research Chair in Biomaterials and V. E. of the University Graduate Fellowship from UBC and the Pacific Century Graduate Scholarship from the Province of British Columbia. We specifically wish to thank Dr. David Embury for most valuable discussions regarding the mechanisms behind the observations. We are also thankful to Dr. Danmei Liu and Dr. Heather McKay from the Centre for Hip Health and Mobility (CHHM) at UBC for discussions and support throughout the project. We are grateful to the UBC Life Science Institute (LSI) Imaging facility for the use of their LSCM.

\section{References}

[1] Martin RB, Burr DB. Structure, Function, and Adaptation of Compact Bone. New York: Raven Press; 1989.

[2] Rho JY, Kuhn-Spearing L, Zioupos P. Mechanical properties and the hierarchical structure of bone. Med Eng Phys 1998;20:92-102.

[3] Weiner S, Wagner HD. The material bone: Structure mechanical function relations. Annu Rev Mater Sci 1998;28:271-98.

[4] Currey JD. Bones: Structure and Mechanics. Princeton, NJ: Princeton Univ. Press; 2002.

[5] Reilly DT, Burstein AH. The elastic and ultimate properties of compact bone tissue. J Biomech 1975;8:393-405.

[6] Zioupos P, Currey JD, Sedman AJ. An examination of the micromechanics of failure of bone and antler by acoustic emission tests and laser scanning confocal microscopy. Med Eng Phys 1994;16:203-12.

[7] Mercer C, He MY, Wang R, Evans AG. Mechanisms governing the inelastic deformation of cortical bone and application to trabecular bone. Acta Biomater 2006;2:59-68.

[8] Ebacher V, Wang R. A unique microcracking process associated with the inelastic deformation of Haversian bone. Adv Funct Mater 2009;19:57-66.

[9] Evans AG, Zok FW. The physics and mechanics of fiber-reinforced brittle matrix composites. J Mater Sci 1994;29:3857-96. 
[10] Wang R, Suo Z, Evans AG, Yao N, Aksay IA. Deformation mechanisms in nacre. J Mater Res 2001;16:2485-93.

[11] Peterlik H, Roschger P, Klaushofer K, Fratzl P. From brittle to ductile fracture of bone. Nat Mater 2006;5:52-5.

[12] Wang R, Gupta HS. Deformation and fracture mechanisms of bone and nacre. Ann Rev Mater Res 2011;41:12.1,12.33 (in press).

[13] Buehler MJ. Molecular nanomechanics of nascent bone: Fibrillar toughening by mineralization. Nanotechnology 2007;18:295102.

[14] Tai K, Dao M, Suresh S, Palazoglu A, Ortiz C. Nanoscale heterogeneity promotes energy dissipation in bone. Nat Mater 2007;6:454-62.

[15] Jager I, Fratzl P. Mineralized collagen fibrils: A mechanical model with a staggered arrangement of mineral particles. Biophys J 2000;79:1737-46.

[16] Gao H, Ji B, Jager IL, Arzt E, Fratzl P. Materials become insensitive to flaws at nanoscale: lessons from nature. Proc Natl Acad Sci U S A 2003;100:5597-600.

[17] Gupta HS, Seto J, Wagermaier W, Zaslansky P, Boesecke P, Fratzl P. Cooperative deformation of mineral and collagen in bone at the nanoscale. Proc Natl Acad Sci U S A 2006;103:17741-6.

[18] Fantner GE, et al. Sacrificial bonds and hidden length dissipate energy as mineralized fibrils separate during bone fracture. Nat Mater 2005;4:612-6.

[19] Fantner GE, Adams J, Turner P, Thurner PJ, Fisher LW, Hansma PK. Nanoscale ion mediated networks in bone: Osteopontin can repeatedly dissipate large amounts of energy. Nano Lett 2007;7:2491-8.

[20] Zioupos P, Currey JD. The extent of microcracking and the morphology of microcracks in damaged bone. J Mater Sci 1994;29:978-86.

[21] Ebacher V, Tang C, McKay H, Oxland TR, Guy P, Wang R. Strain redistribution and cracking behavior of human bone during bending. Bone 2007;40:1265-75.

[22] Zioupos P, Hansen U, Currey JD. Microcracking damage and the fracture process in relation to strain rate in human cortical bone tensile failure. J Biomech 2008;41:2932-9.

[23] Carter DR, Hayes WC. Compact bone fatigue damage: a microscopic examination. Clin Orthop Relat Res 1977;(127):265-74.

[24] Reilly GC, Currey JD. The development of microcracking and failure in bone depends on the loading mode to which it is adapted. J Exp Biol 1999;202:543-52. 
[25] Reilly GC. Observations of microdamage around osteocyte lacunae in bone. J Biomech 2000;33:1131-4.

[26] Burr DB, Turner CH, Naick P, Forwood MR, Ambrosius W, Hasan MS, Pidaparti R. Does microdamage accumulation affect the mechanical properties of bone? J Biomech 1998;31:33745 .

[27] O'Brien FJ, Taylor D, Clive Lee T. The effect of bone microstructure on the initiation and growth of microcracks. J Orthop Res 2005;23:475-80.

[28] Skedros JG, Holmes JL, Vajda EG, Bloebaum RD. Cement lines of secondary osteons in human bone are not mineral-deficient: new data in a historical perspective. Anat Rec A Discov Mol Cell Evol Biol 2005;286:781-803.

[29] Norman TL, Wang Z. Microdamage of human cortical bone: incidence and morphology in long bones. Bone 1997;20:375-9.

[30] Koester KJ, Ager III JW, Ritchie RO. The true toughness of human cortical bone measured with realistically short cracks. Nat Mater 2008;7:672.

[31] Nalla RK, Kinney JH, Ritchie RO. Mechanistic fracture criteria for the failure of human cortical bone. Nat Mater 2003;2:164-8.

[32] Fantner GE, et al. Hierarchical interconnections in the nano-composite material bone: Fibrillar cross-links resist fracture on several length scales. Composites Sci Technol 2006;66:1205-11.

[33] Vashishth D. Hierarchy of bone microdamage at multiple length scales. Int J Fatigue 2007;29:1024-33.

[34] Ebacher V, Wang R. Circumferential arc-shaped microcracks in Haversian bone: LeadUranyl acetate staining for micro-CT imaging, in Structure-Property Relationships in Biomineralized and Biomimetic Composites. Mater Res Soc Symp Proc 2009;1187:141-6.

[35] You LD, Weinbaum S, Cowin SC, Schaffler MB. Ultrastructure of the osteocyte process and its pericellular matrix. Anat Rec A Discov Mol Cell Evol Biol 2004;278:505-13.

[36] Currey JD. The many adaptations of bone. J Biomech 2003;36:1487-95.

[37] Ascenzi A, Bonucci E. Mechanical similarities between alternate osteons and cross-ply laminates. J Biomech 1976;9:65-71.

[38] Parsamian GP, Norman TL. Diffuse damage accumulation in the fracture process zone of human cortical bone specimens and its influence on fracture toughness. J Mater Sci -Mater Med 2001;12:779-83. 
[39] Ascenzi A, Benvenuti A. Orientation of collagen fibers at the boundary between 2 successive osteonic lamellae and its mechanical interpretation. J Biomech 1986;19:455-63.

[40] Reid SA. A study of lamellar organisation in juvenile and adult human bone. Anat Embryol 1986;174:329-38.

[41] Kazanci M, Wagner HD, Manjubala NI, Gupta HS, Paschalis E, Roschger P, Fratzl P. Raman imaging of two orthogonal planes within cortical bone. Bone 2007;41:456-61.

[42] Green M, Isaac DH, Jenkins GM. Collagen fibre orientation in bovine secondary osteons by collagenase etching. Biomaterials 1987;8:427-32.

[43] Voide R, et al. Time-lapsed assessment of microcrack initiation and propagation in murine cortical bone at submicrometer resolution. Bone 2009;45:164-73.

[44] Nicolella DP, Moravits DE, Gale AM, Bonewald LF, Lankford J. Osteocyte lacunae tissue strain in cortical bone. J Biomech 2006;39:1735-43.

[45] McVeigh C, Vernerey F, Liu WK, Moran B, Olson G. An interactive micro-void shear localization mechanism in high strength steels. J Mech Phys Solids 2007;55:225-44.

[46] Jepsen KJ, Davy DT, Krzypow DJ. The role of the lamellar interface during torsional yielding of human cortical bone. J Biomech 1999;32:303-10.

[47] Burr DB, Martin RB, Schaffler MB, Radin EL. Bone remodeling in response to in vivo fatigue microdamage. J Biomech 1985;18:189-200.

[48] Schaffler MB, Radin EL, Burr DB. Mechanical and morphological effects of strain rate on fatigue of compact bone. Bone 1989;10:207-14.

[49] Schaffler MB, Choi K, Milgrom C. Aging and matrix microdamage accumulation in human compact bone. Bone 1995;17:521-5.

[50] Lee TC, O'Brien FJ, Taylor D. The nature of fatigue damage in bone. Int J Fatigue 2000;22:847-53.

[51] Cowin SC, Hegedus DH. Bone remodeling I: Theory of adaptive elasticity. J Elast 1976;6:313-26.

[52] Frost HM. Presence of microscopic cracks in vivo in bone. Henry Ford Hosp Med Bull 1960;27-35.

[53] Martin RB, Burr DB. A hypothetical mechanism for the stimulation of osteonal remodeling by fatigue damage. J Biomech 1982;15:137-9. 
[54] Bentolila V, Boyce TM, Fyhrie DP, Drumb R, Skerry TM, Schaffler MB. Intracortical remodeling in adult rat long bones after fatigue loading. Bone 1998;23:275-81.

[55] Verborgt O, Gibson GJ, Schaffler MB. Loss of osteocyte integrity in association with microdamage and bone remodeling after fatigue in vivo. J Bone Miner Res 2000;15:60-7.

[56] Piekarski K, Munro M. Transport mechanism operating between blood supply and osteocytes in long bones. Nature 1977;269:80-2.

[57] Weinbaum S, Cowin SC, Zeng Y. A model for the excitation of osteocytes by mechanical loading-induced bone fluid shear stresses. J Biomech 1994;27:339-60.

[58] Burger EH, Klein-Nulend J. Mechanotransduction in bone - role of the lacuno-canalicular network. FASEB J 1999;13:S101-12.

[59] Han YF, Cowin SC, Schaffler MB, Weinbaum S. Mechanotransduction and strain amplification in osteocyte cell processes. Proc Natl Acad Sci U S A 2004;101:16689-94.

[60] Qiu SJ, Rao DS, Fyhrie DP, Palnitkar S, Parfitt AM. The morphological association between microcracks and osteocyte lacunae in human cortical bone. Bone 2005;37:10-5.

[61] Hazenberg JG, Freeley M, Foran E, Lee TC, Taylor D. Microdamage: a cell transducing mechanism based on ruptured osteocyte processes. J Biomech 2006;39:2096-103.

[62] Busse B, et al. Decrease in the osteocyte lacunar density accompanied by hypermineralized lacunar occlusion reveals failure and delay of remodeling in aged human bone. Aging Cell 2010;9:1065-75.

[63] Ascenzi M, Gill J, Lomovtsev A. Orientation of collagen at the osteocyte lacunae in human secondary osteons. J Biomech 2008;41:3426-35.

[64] Gonzalez C, LLorca J. Mechanical behavior of unidirectional fiber-reinforced polymers under transverse compression: Microscopic mechanisms and modeling. Composites Sci Technol 2007;67:2795-806.

[65] Ascenzi A, Bonucci E, Simkin A. An approach to the mechanical properties of single osteonic lamellae. J Biomech 1973;6:227-35.

[66] Braidotti P, Branca FP, Stagni L. Scanning electron microscopy of human cortical bone failure surfaces. J Biomech 1997;30:155-62.

[67] Gupta HS, Zioupos P. Fracture of bone tissue: The 'hows' and the 'whys'. Med Eng Phys 2008;30:1209-26.

[68] Ashby MF, Hallam SD. The failure of brittle solids containing small cracks under compressive stress states. 1986;34:497-510. 
[69] Zioupos P. Accumulation of in-vivo fatigue microdamage and its relation to biomechanical properties in ageing human cortical bone. J Microsc 2001;201:270-8.

[70] Diab T, Vashishth D. Morphology, localization and accumulation of in vivo microdamage in human cortical bone. Bone 2007;40:612-8.

[71] Schaffler MB, Pitchford WC, Choi K, Riddle JM. Examination of compact bone microdamage using back-scattered electron microscopy. Bone 1994;15:483-8. 


\section{Figures}

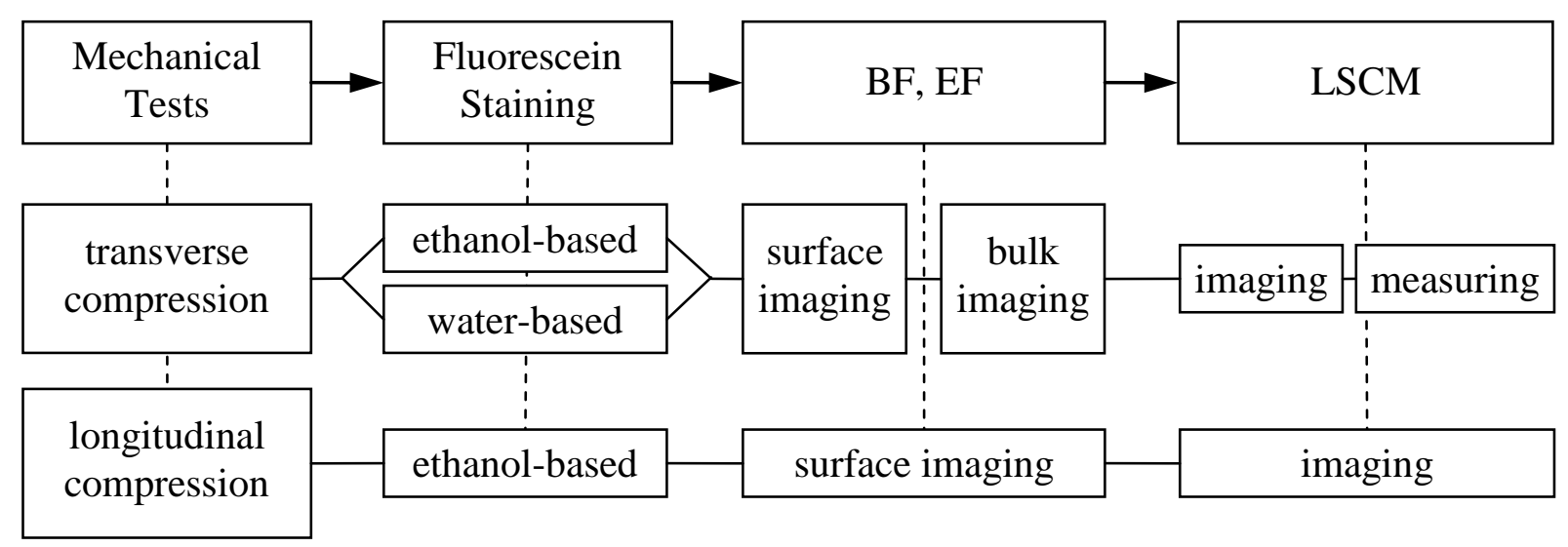

Fig. 1. Summary of experimental procedures. A total of 17 human cortical bone specimens were submitted to compressive loading along two different orientations. For each orientation, 2 specimens were loaded to fracture and 5 specimens were unloaded prior to fracture (i.e., at about 5\% drop from peak load). The latter were also used to count the proportions of damaged osteons. For the transverse orientation, 3 additional specimens were loaded in a step-wise manner to monitor deformation and damage development under BF and EF (see [8] for details). All specimens were stained in an ethanolbased fluorescein solution except for one which was kept fresh (non-dehydrated, stained in a water-based solution of fluorescein, and imaged under water immersion). All specimens surfaces were examined under $\mathrm{BF}$ and $\mathrm{EF}$ and 4 specimens were subsequently ground and polished to their central section to examine microcracking within the bulk. LSCM imaging followed and images from 7 transversely compressed specimens were used to characterize the canaliculi and cracks distributions. Two regions (or images) per specimen were analyzed for a total of fourteen regions. 

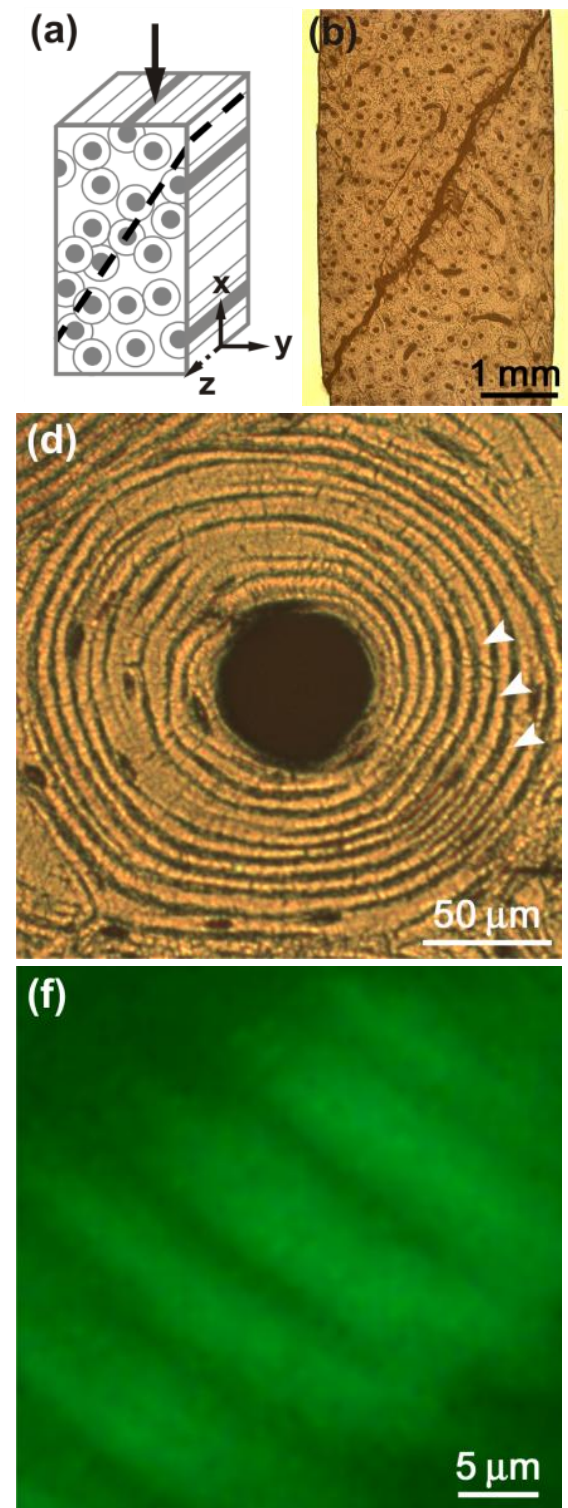

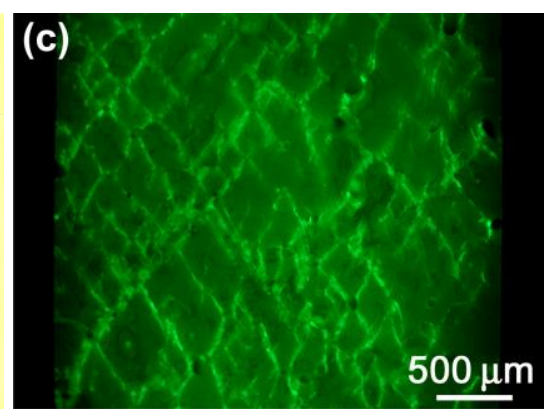

(e)
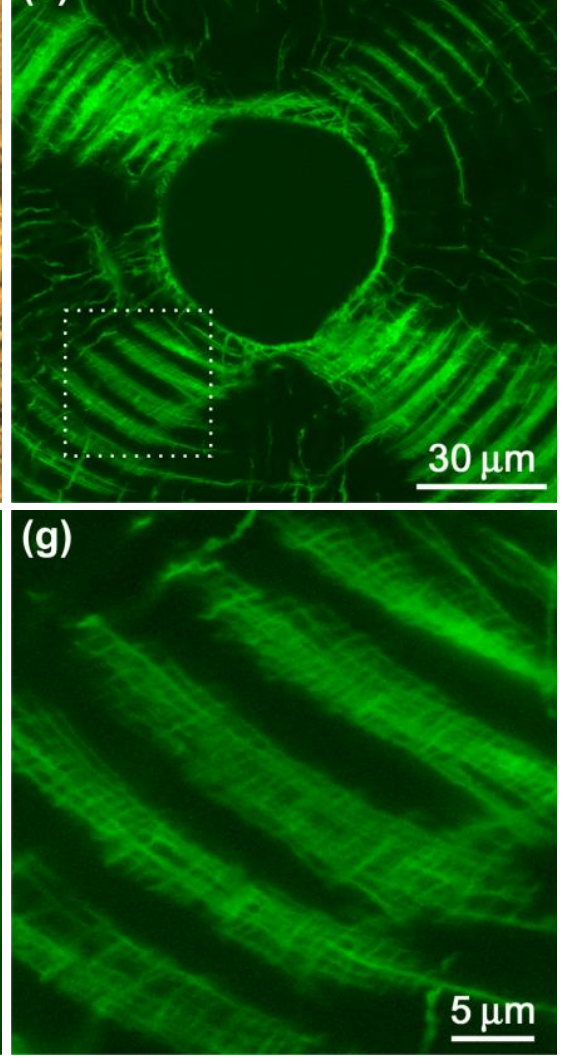

Fig. 2. Multi-scale microcracking in human cortical bone under transverse compression. (a) Schematics of the transverse $\left(90^{\circ}\right)$ loading orientation with respect to the osteons; (b) BF image of the macroscopic oblique fracture pattern; (c) EF image of distributed cross-hatched damage at the osteonal-interstitial level; (d) BF image of arc-shaped microcracks within the osteonal bright layers (arrowheads); (e) LSCM image showing multiple intralamellar cracks in the four quadrants of an osteon; (f-g) Low resolution EF and high resolution LSCM images taken from the lower left quadrant (dotted line) of the osteon in (e). LSCM reveals a sub-lamellar cross-hatched pattern composed of fine radially and circumferentially oriented cracks with spacing similar in size to fibrillar bundles. Compressive load applied vertically for ab-c and horizontally for d-e-f-g. 

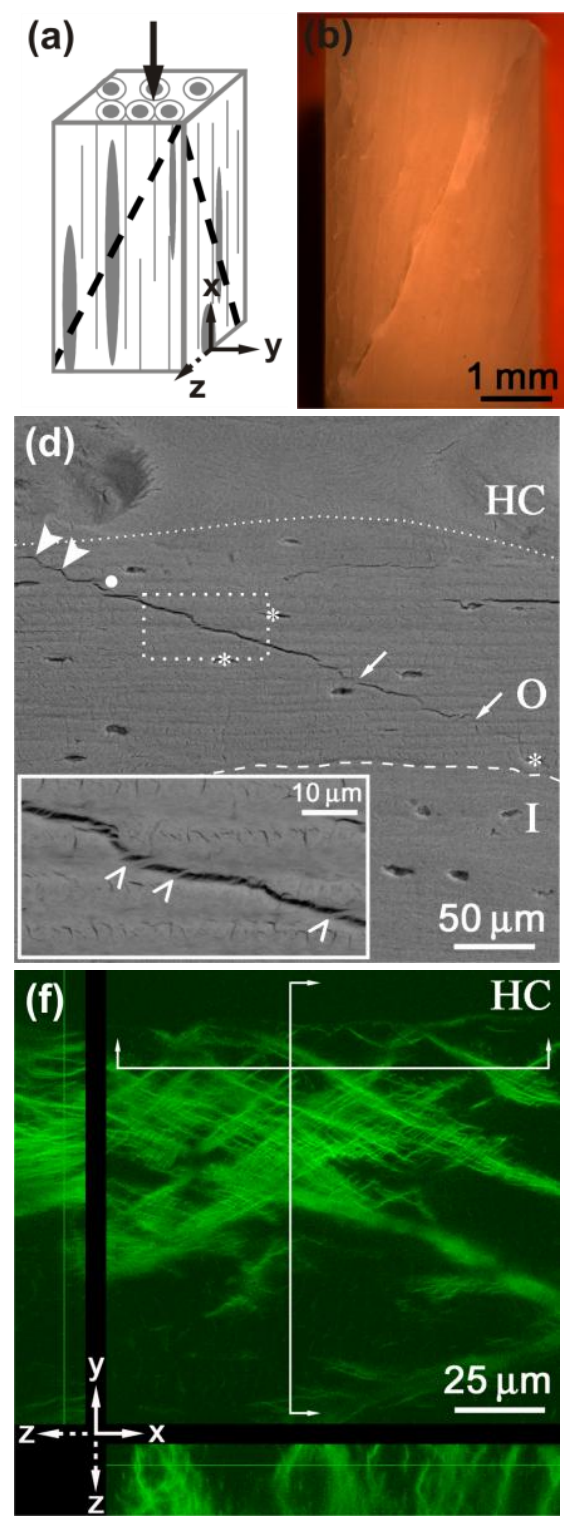
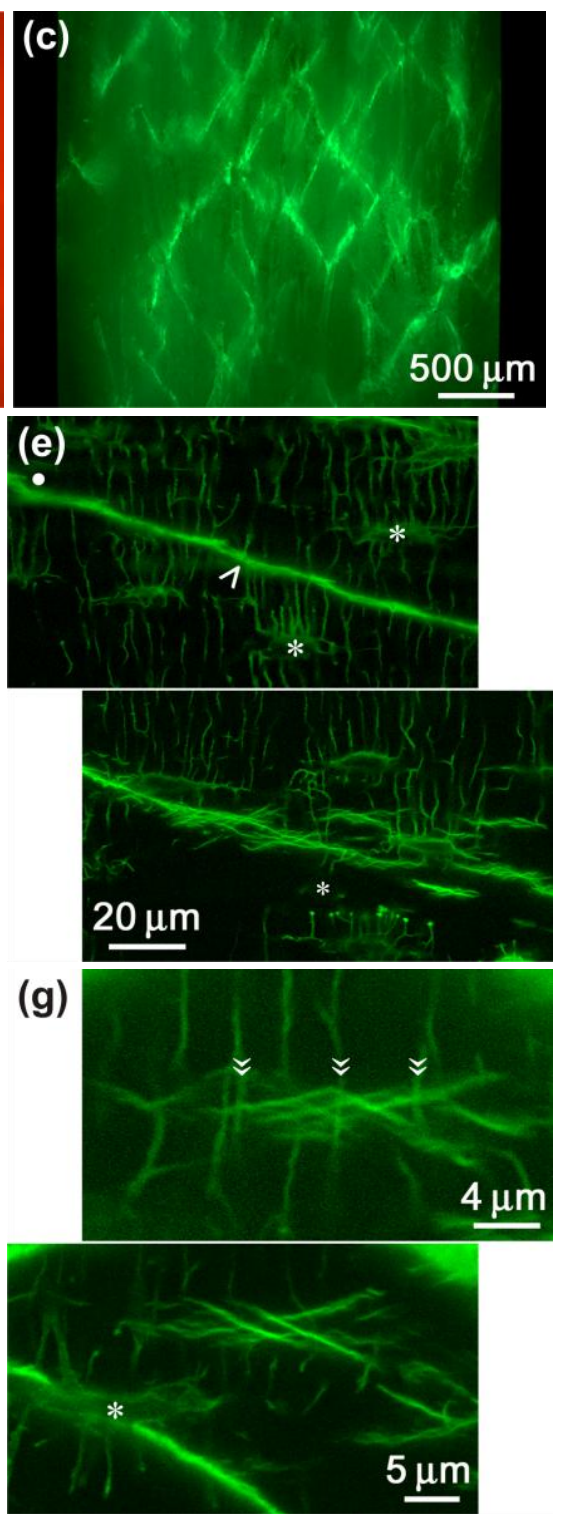

Fig. 3. Multi-scale microcracking in human cortical bone under longitudinal compression. (a) Schematics of the longitudinal $\left(0^{\circ}\right)$ loading orientation with respect to the osteons; (b) Stereomicroscope image of the macroscopic oblique fracture pattern; (c) EF image of distributed cross-hatched damage at the osteonalinterstitial level. Note the morphological similarity with transverse compression damage; (d) Backscattered electron (BSE) micrograph of osteonal oblique microcrack formed at the Haversian canal (HC; approximated by dotted line) and extending to the boundary (approximated by dashed line) between osteonal (O) and interstitial (I) bone. Notice the "stairway-like" changes of orientations (arrowheads) with the layers as well as the uncracked ligaments (insert: empty arrowheads) and lamellae (arrows). Insert location shown by dotted line; (e) LSCM images showing smaller cracks with the larger oblique microcrack. The identified osteocyte lacunae (asterisks), ligament bridging (empty arrowheads) and full circle correspond to the same locations in (d). Note the change in crack morphology near the osteonalinterstitial boundary (bottom image); (f) High resolution 3D LSCM image of localized, finely spaced cross-hatched cracking near a Haversian canal (HC). The z-planes ("cut views" at locations shown by white lines) show deep oblique cracking; (g) LSCM images of crack interactions with osteocyte lacunae (asterisk) and canaliculi (double arrows). Compressive load applied vertically for a-b-c and horizontally for d-e-f-g. 

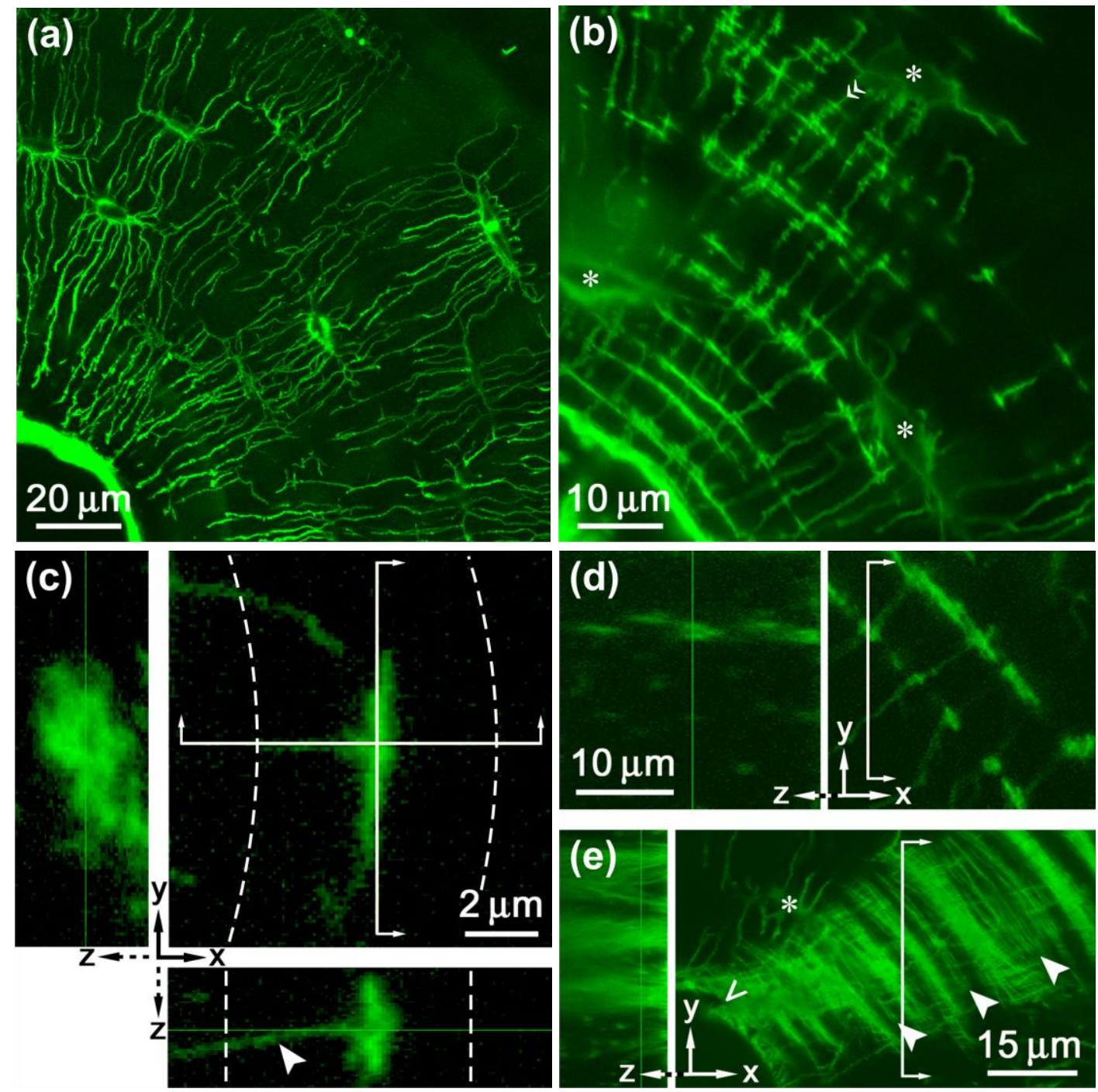

Fig. 4. LSCM imaging of the canalicular network and the intralamellar cracking sequence within the bulk of transversely compressed bone specimens. (a) Image of a $5 \mu \mathrm{m}$ deep z-stack showing the lacunocanalicular network within an intact osteon; (b) Multiple circumferential crack initiations at the canaliculi. A single canaliculus could initiate more than one crack (double arrow). Osteocyte lacunae are identified by asterisks; (c) Views (xy, xz, and yz) of a single crack associated with a canaliculus (arrowhead). Dashed lines are approximated lamellar boundaries; (d) Merging process of the circumferential cracks in the circumferential and depth (z-plane) directions; (e) Bright and dark (arrowheads) layers damage associated with the formation of a shear band. The microcracks have a "sheet-like" appearance through the depth (z-plane). Note also the radially oriented microcrack at the Haversian canal (empty arrowhead). Compressive load applied horizontally for b-d-e and obliquely for $\mathrm{c}$. 

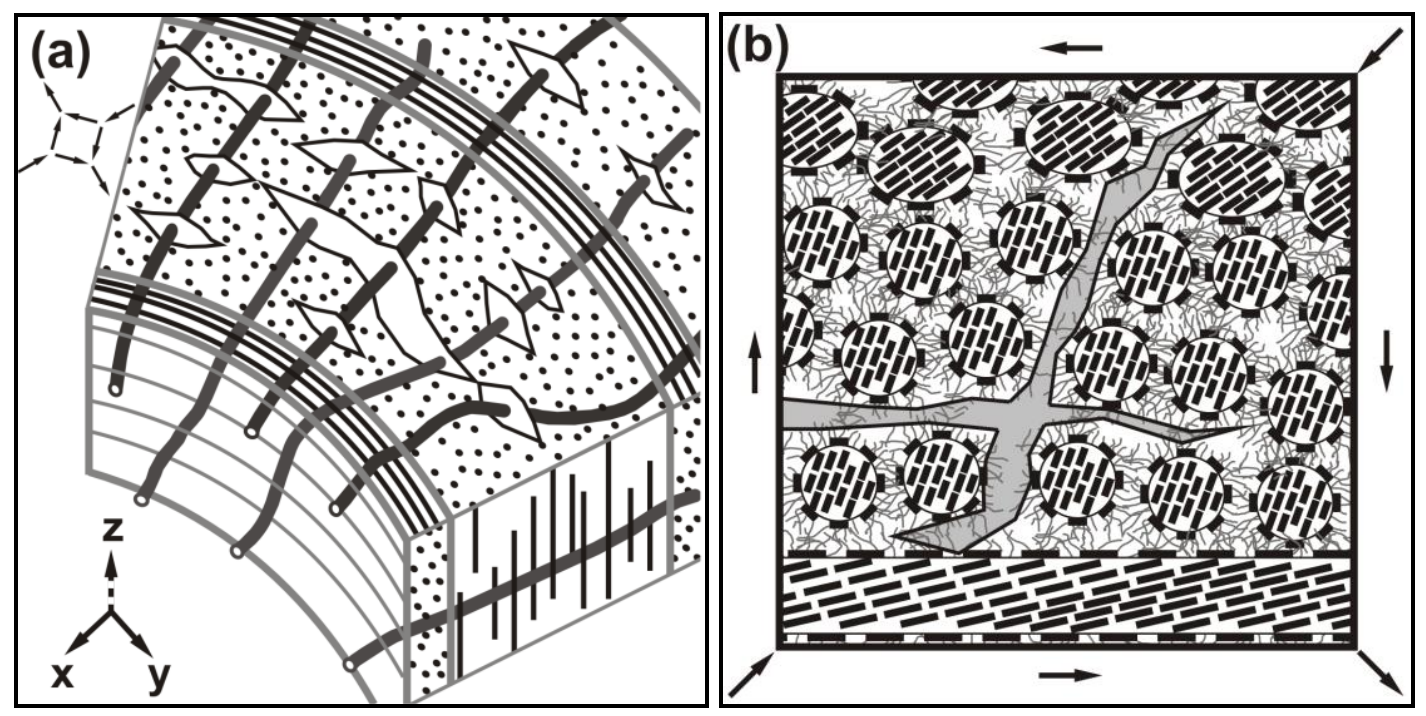

Fig. 5. Physical interpretation of sub-lamellar structure - microcracking relations in bone under transverse compression. (a) Schematic of multiple circumferential crack nucleations and mergings at the radially oriented canaliculi (x-direction) under interlamellar shear (stress state depicted) in osteonal layers with fibrils oriented parallel to the osteons; (b) Proposed interfibrillar nature of the sub-lamellar cracks (light grey) with respect to the rotating mineralized collagen fibrils (minerals in black) and the surrounding noncollagenous proteins (small grey lines). Arrows indicate the stress state depicted in (a). 